\title{
镁卟啉与氮、氧杂环化合物的相互作用
}

\author{
许惠英 ${ }^{1, *}$ 王 维 ${ }^{2}$ \\ ('浙江树人大学生物与环境工程学院, 杭州 310015; 2镇海环境监测站, 浙江 宁波 315200)
}

\begin{abstract}
摘要: 以镁卟啉为主体化合物, 模拟了生物体内常见的氮、氧杂环客体与主体化合物之间的相互作用. 研究结 果表明: 镁卟啉与氮、氧杂环化合物的相互作用引起了镁卟啉中的镁原子与卟吩环不共面, 且二面角越小不共 面程度越大. 自然键轨道(NBO)理论分析表明氮、氧孤对电子和金属镁的空孤对轨道的相互作用对复合物的稳 定性贡献很大. 使用约化密度梯度(RDG)函数等值面图和散点图可视化分析了配位相互作用和周边氢键作用 的位置及强度. 概念密度泛函(DFT)理论参数表明所形成的复合物比主体化合物的热力学稳定性小而反应活性 高. 芳香性计算表明含氧杂环客体与主体的相互作用使复合物中的卟吩环具有反芳香性, 而含氮杂环客体与主 体的相互作用使复合物中卟吩环呈现区域性芳香性.
\end{abstract}

关键词：镁卟啉； 约化密度梯度函数等值面图；配位相互作用；自然键轨道理论；芳香性 中图分类号: 0641

\section{Interaction between Mg-Porphyrin and Nitrogen, Oxygen Heterocyclic Compounds}

\author{
XU Hui-Ying ${ }^{1, *} \quad$ WANG Wei ${ }^{2}$ \\ $\left({ }^{1}\right.$ College of Biology \& Environment Engineering, Zhejiang Shuren University, Hangzhou 310015, P. R. China; \\ ${ }^{2}$ Zhenhai Environmental Monitoring Station, Ningbo 315200, Zhejiang Province, P. R. China)
}

\begin{abstract}
The interaction between nitrogen-oxygen heterocyclic compounds and a host (magnesium porphyrin) was investigated. The results showed that the magnesium atoms in the magnesium porphyrin and porphin ring were not coplanar and at a smaller dihedral angle the extent of its non-coplanar nature increased. Natural bond orbital (NBO) analysis indicated that the interaction between the lone pair of electrons on the nitrogen and oxygen atoms and the unoccupied lone pair orbital of magnesium contributes significantly to the stability of the complexes. The reduced density gradient (RDG) isosurface map and the scatter diagram indicated the location and intensity of the axial coordination interactions and the surrounding hydrogen bonding interactions. The conceptual density functional theory (DFT) parameter indicated that the complex compounds are less thermodynamically stable than magnesium porphyrin, however, they have higher reactivity. Aromatic calculations revealed that the interaction between the oxygen-containing heterocycles and the host compound made the porphin ring in the complexes anti-aromatic while the interaction between the nitrogen-containing heterocyclic and the host made the porphin ring in the complexes regionally aromatic.
\end{abstract}

Key Words: Magnesium porphyrin; RDG iso-surface map; Coordination interaction; Natural bond orbital; Aromaticity

Received: July 4, 2011; Revised: September 4, 2011; Published on Web: September 15, 2011.

"Corresponding author. Email: xuhy65@163.com; Tel: +86-571-88299821.

The project was supported by the Program of Education Department of Zhejiang Province, China (Y200803060).

浙江省教育厅科研项目(Y200803060)资助

(C) Editorial office of Acta Physico-Chimica Sinica 


\section{1 引 言}

卟啉是在卟吩环上带有取代基的一类大环化 合物总称. 卟吩是由四个吡咯环和四个次甲基桥联 起来的大 $\pi$ 共轭体系. 由于卟啉和金属卟啉在自然 界和生命体中广泛存在, 所以受到理论和实验学家 的关注. ${ }^{1,2}$ 在催化(包括均相、多相催化及电催化, 光 催化等)、 ${ }^{3-5}$ 谱学性质、 ${ }^{6}$ 非线性光学材料、 ${ }^{7}$ 分子器 件、 ${ }^{8}$ 色谱固定相材料 ${ }^{9}$ 等诸多领域, 卟啉和金属卟啉 有着广阔的应用前景. 叶绿素广泛存在于绿色植物 中, 是绿色植物以光合作用方式将太阳能转化成化 学能从而维系自身新陈代谢的主要化合物, 其核心 结构是吓啉的金属配合物即镁卟啉. ${ }^{10,11}$ 因此, 揭示 镁卟啉与客体分子之间的相互作用, 对研究生物体 内传输、诱导转换具有重要理论指导意义. 本文以 片段截取的方法选取了生物分子中常见的氮、氧杂 环小分子基团为客体分子(如吡咯分子), 采用量子 化学模型, 研究镁卟啉与杂环化合物的相互作用, 以期为生命过程化学本质研究提供重要的线索.

\section{2 计算方法}

用 Gaussian $03^{12}$ 中的 B3LYP/6-311G**基组, 对 镁卟啉主体及客体分别进行几何构型优化, 由于复 合物都为前三周期原子, 且分子体系较大, 又属于 弱相互作用, 故复合物采用 B3LYP/6-311G**优化. 用 B3LYP/6-311G** 优化的主体构型构建复合物, 镁卟啉与 6 个氮、氧杂环化合物以 $1: 1$ 形成的超分子 复合物见图 1. 初始模型 ${ }^{13}$ 的构建如下: (a) 客体分子 轴向垂直于镁卟啉平面; (b) 客体分子与镁卟啉最短 分子间距为 $0.45 \mathrm{~nm}$; (c) 客体分子中心与镁卟啉中 心距离为 $0.65 \mathrm{~nm}$. 用 B3LYP/6-311G $\mathrm{G}^{* *}$ 方法对复合 物 I-VI 进行几何构型全优化, 不加任何对称性限 制. 将复合物看作一个整体, 即电子在整个超分子 轨道中运动, 则可以找到势能面上的能量极小值. 复合物的能量减去主体和客体单独存在时的能量 即为复合物的稳定化能 $(\Delta E)$. 对七个复合物稳定化 能采用 Boys-Bemardi 方法进行校正..$^{14}$ 用 Gaussian 03 中 3.1 版本的 NBO 程序计算二级微扰稳定化能 $\left(\Delta E^{2}\right)$. 在 B3LYP/6-311G**优化构型基础上, 用规范 无关原子轨道 $(\mathrm{GIAO})$ 方法在 B3LYP/6-31++ $\mathrm{G}^{* *}$ 水 平上进行 ${ }^{3} \mathrm{He}$ 化学位移计算. 氦原子单独存在时的 化学位移与此值之差即为核无关化学位移(NICS)数 值. 在 B3LYP/6-311G**优化构型上, 以复合物 I 的

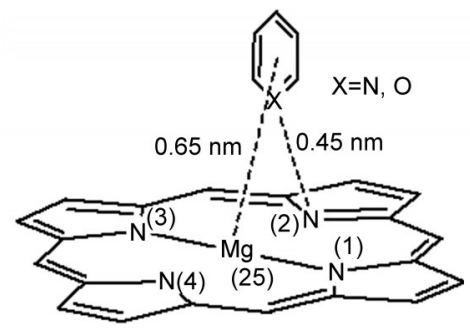

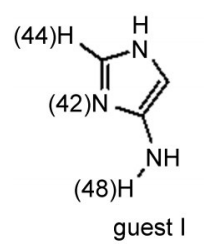

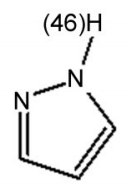

guest II

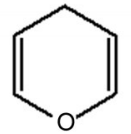

guest IV

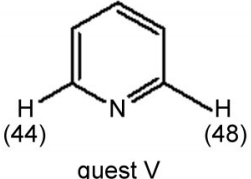

guest $\mathrm{V}$
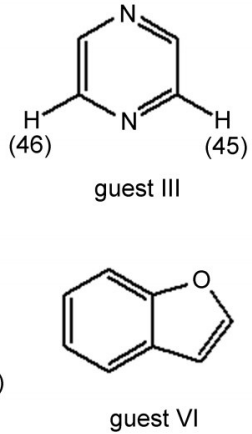

图 1 主-客体初始构型
Fig.1 Host-guest initial configuration

$\mathrm{N}(42)$ 和 $\operatorname{Mg}(25)$ 中点为中心, 向四周延展, 各方向格 点数为 $60,60,60$, 来计算空间内各点的 RDG 函数 和 $\operatorname{sign}\left(\lambda_{2}(r)\right) \rho(r)$ 函数的值, ${ }^{15}$ 用 VMD 程序进行 $\mathrm{RDG}$ 等值面图的绘制.

\section{3 结果与讨论}

\section{1 几何结构与相互作用能分析}

表 1 给出采用 B3LYP/6-311G**方法所得到镁 卟啉与六个复合物分子的几何参数和相互作用能. 表中复合物未校正的稳定化能 $(\Delta E)$ 及基组重叠误差 (BSSE) 校正过的稳定化能 $(\Delta E+\mathrm{BSSE})$ 均为负值, 说 明二者所形成复合物为稳定构型. 由表 1 中的二面 角数据可以看出主体分子中的镁原子与卟吩环共 平面, 而优化后的复合物分子由于存在配位作用和 周边氢键作用(本文所指周边氢键为杂环客体上的 氢原子与镁卟啉中的氮原子所形成的氢键), 导致镁 卟啉主体的几何结构发生扭转, 镁原子与卟吩环不 共面, 并且镁原子与卟吩环中的氮原子距离变长. 表 1 中的数据说明配位作用和周边氢键相互作用的 强度不同, 对六个复合物二面角的影响也各不相 同, 但总体上表现出这样的规律: 复合物的稳定化 能越大, 其二面角越小.

图 2 中列出了具有代表性的氮、氧杂环客体与 镁卟啉相互作用后的构型优化图, 从图中可以看出 氮、氧杂环客体分子以不同的结合方式与镁卟啉形 
表 1 B3LYP/6-311G**水平上复合物和主体的几何参数与稳定化能

Table 1 Geometric parameters and stabilization energy of complexes and host calculated at the B3LYP/6-311G** level

\begin{tabular}{cccccccc}
\hline Complex & $\angle \mathrm{N}(4) \mathrm{N}(3) \mathrm{Mg}(25) \mathrm{N}(2) /\left(^{\circ}\right)$ & $\angle \mathrm{N}(3) \mathrm{N}(4) \mathrm{Mg}(25) \mathrm{N}(1) /\left(^{\circ}\right)$ & $d_{\mathrm{Mg}-\mathrm{N}, \mathrm{o}} / \mathrm{nm}$ & Hydrogen bond & $d / \mathrm{nm}$ & $\Delta E / \mathrm{eV}$ & $(\Delta E+\mathrm{BSSE}) / \mathrm{eV}$ \\
\hline host & 199.9 & 180.0 & - & & & - & - \\
I & 159.4 & 158.0 & 0.222 & $\mathrm{H}(48) \cdots \mathrm{N}(2)$ & 0.234 & -1.293 & -1.140 \\
& & & & $\mathrm{H}(44) \cdots \mathrm{N}(4)$ & 0.272 & & \\
$\mathrm{II}$ & 163.5 & 160.8 & 0.222 & $\mathrm{H}(46) \cdots \mathrm{N}(2)$ & 0.239 & -1.158 & -1.023 \\
III & 163.9 & 162.8 & 0.227 & $\mathrm{H}(46) \cdots \mathrm{N}(4)$ & 0.270 & -1.040 & -0.912 \\
& & & & $\mathrm{H}(45) \cdots \mathrm{N}(2)$ & 0.270 & & -0.691 \\
IV & 165.9 & 165.9 & 0.224 & - & & -0.807 & -0.974 \\
V & 161.8 & 160.7 & 0.225 & $\mathrm{H}(48) \cdots \mathrm{N}(2)$ & 0268 & -1.102 & \\
& & & & $\mathrm{H}(44) \cdots \mathrm{N}(4)$ & 0.268 & & -0.653 \\
VI & 166.0 & 166.0 & 0.231 & - & & -0.763 & -0.63 \\
\hline
\end{tabular}

$\Delta E$ : stabilization energy; $\Delta E+\mathrm{BSSE}$ : BSSE corrected stabilization energy; $d_{\mathrm{Mg}-\mathrm{N}, \mathrm{o}}$ : coordinate bond distance; $d$ : hydrogen bond distance

成稳定的复合物, 表 1 中的数据可以看出复合物稳 定化能由配位作用和周边氢键相互作用共同影响. 不难发现六个复合物中含氮杂环化合物与美卟啉 在形成配位作用的同时还存在周边氢键相互作用, 而含氧杂环客体与镁卟啉只形成配位作用并未形 成氢键相互作用, 这也就意味着含氧杂环化合物比 含氮杂环化合物稳定化能小, 说明在生物体内的配 位-诱导分子转换过程中含氧杂环客体容易被含氮 杂环客体所诱导从而被置换.

\subsection{NBO 分析}

自然键轨道理论(NBO)可以从轨道相互作用的 角度来理解复合物的形成过程. ${ }^{16}$ 由轨道相互作用 引起的能量稳定可以用二级微扰稳定化能 $\left(\Delta E^{2}\right)$ 来 描述. 二级微扰稳定化能 $\left(\Delta E^{2}\right)$ 可以由下式得到

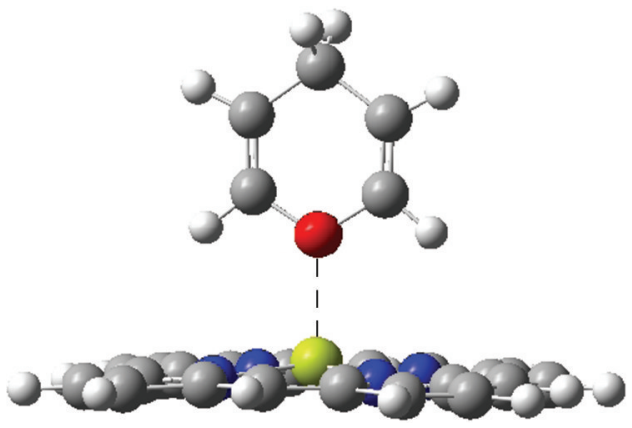

$$
\Delta E^{2}=\Delta E_{i j}=q_{i} \frac{F(i, j)^{2}}{\varepsilon_{j}-\varepsilon_{i}}
$$

其中 $i$ 为电子供体轨道, $j$ 为电子受体轨道, $q_{i}$ 为供体 轨道的占据数, $\varepsilon_{i}$ 和 $\varepsilon_{j}$ 为对角元素 (轨道能量), $F$ 为 NBO Fock矩阵元. 表 2 列出了氮、氧杂环客体与镁 卟啉形成配位复合物过程中配位相互作用中主要 的二级微扰稳定化能 $\Delta E^{2}$ 和供体轨道占据数 $\left(q_{i}\right)$ 、受 体轨道的占据数 $\left(q_{j}\right)$.

$\mathrm{NBO}$ 分析结果见表 2, 表 2 中列出了供体轨道 $(i)$ 和受体轨道 $(j)$ 的占据数, 供-受轨道的占据数说明: 供 体轨道为 $\mathrm{X}(\mathrm{N} 、 \mathrm{O})$ 的孤对电子 (LP) 其占据数约 1.90 , 受体轨道为 $\mathrm{Mg}$ 的孤对轨道( $\mathrm{LP}$ )或空孤对轨道 $\left(\mathrm{LP}^{*}\right)$ 其占据数约为 0.23 . 这里特别指出 Gaussian 03 程序 输出复合物 I、IV、VI中 Mg 的受体轨道为孤对轨道

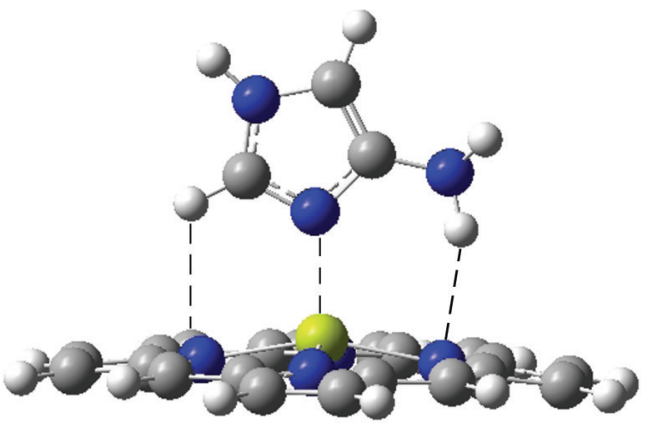

图 $2 \mathrm{~B} 3 \mathrm{LYP} / 6-311 \mathrm{G} * *$ 水平上复合物 I 和 IV 的优化构型

Fig.2 Optimized geometries of complexes I and IV at the B3LYP/6-311G** level

表 2 在 B3LYP/6-311G**水平上复合物 I-VI NBO 分析

Table 2 Natural bond orbital analysis of complexes I-VI at the B3LYP/6-311G** level

\begin{tabular}{cccccc}
\hline Complex & Donor NBO & $q_{i}$ & Acceptor NBO & $q_{j}$ & $\Delta E^{2} /\left(\mathrm{kJ} \cdot \mathrm{mol}^{-1}\right)$ \\
\hline I & LP(1)N & 1.90260 & LP(1)Mg & 0.23771 & 91.00 \\
II & LP(1)N & 1.91927 & LP*(1)Mg & 0.23801 & 90.21 \\
III & LP(1)N & 1.90909 & LP*(1)Mg & 0.23393 & 70.87 \\
IV & LP(1)O & 1.94962 & LP(1)Mg & 0.22661 & 50.57 \\
V & LP(1)N & 1.90583 & LP*(1)Mg & 0.23374 & 75.77 \\
VI & LP(1)O & 1.95570 & LP(1)Mg & 0.22687 & 44.96 \\
\hline
\end{tabular}

$q_{i}$ : donor orbital occupancy; $q_{j}$ : acceptor orbital occupancy; $\Delta E^{2}$ : second order perturbation energy 
(LP), 但由于占据数较低, 被认为是空孤对轨道 $\left(L P^{*}\right)$. 六个复合物配位作用中主要稳定因素为客体 分子的氮、氧孤对电子和金属镁的空孤对轨道即 $L P(1) X \rightarrow L P *(1) M g$ 之间的作用. 并且, 我们发现该 二级微扰稳定化能与复合物的相互作用能高度相 关, 相关系数为 0.98 . 图 3 显示的是这个线性关系 图. 根据这些结果, 可以推断 $\mathrm{LP}(1) \mathrm{X} \rightarrow \mathrm{LP} *(1) \mathrm{Mg}$ 轨 道相互作用对复合物的稳定性贡献很大.

\subsection{RDG 分析}

Yang 课题组 ${ }^{17}$ 提出了一种弱相互作用的可视化 研究方法. 该方法以空间上各点的约化密度梯度 $(\mathrm{RDG})$ 函数和 $\operatorname{sign}\left(\lambda_{2}(r)\right) \rho(r)$ 函数的计算值, 做可视 化的 RDG 等值面图, 以便直观地了解到分子中哪些 区域与弱相互作用有关. 其中 RDG 函数的表达式 为 $\operatorname{RDG}(r)=\frac{1}{2\left(3 \pi^{2}\right)^{1 / 3}} \frac{|\nabla \rho(r)|}{\rho(r)^{4 / 3}}$, 函数中包含了原子中 的分子理论(AIM)中弱相互作用临界点的 $\rho(r), \rho(r)$ 是衡量弱相互作用强度的重要指标之一, 其数值和 键的强度存在正相关性. $\rho(r)$ 不足之处在于它只能 反映出强度不能反映类型, 所以由 $\operatorname{sign}\left(\lambda_{2}(r)\right)$ 函数来 反映类型, 这个函数是电子密度 Hessian矩阵的第二 大的本征值 $\lambda_{2}$ 的符号. 可以将 $\operatorname{sign}\left(\lambda_{2}(r)\right)$ 函数用不同 色彩投影到 RDG 等值面上, 用来表现某一个区域的 相互作用类型. 若将 $\rho(r)$ 和 $\operatorname{sign}\left(\lambda_{2}(r)\right)$ 函数相乘而得 的 $\operatorname{sign}\left(\lambda_{2}(r)\right) \rho(r)$ 函数投影到 RDG 等值面上, 则弱相 互作用的位置、强度、类型都能一目了然地显现出来. 也就是定义一个实空间函数, 使其数值能够区分开体 系中具有不同特征的区域. 最近 Zou 等 ${ }^{18}$ 将该方法 应用于生物体内卤键的研究, 本文以复合物 I 为例 将该方法应用于配位相互作用研究, 验证其在不同

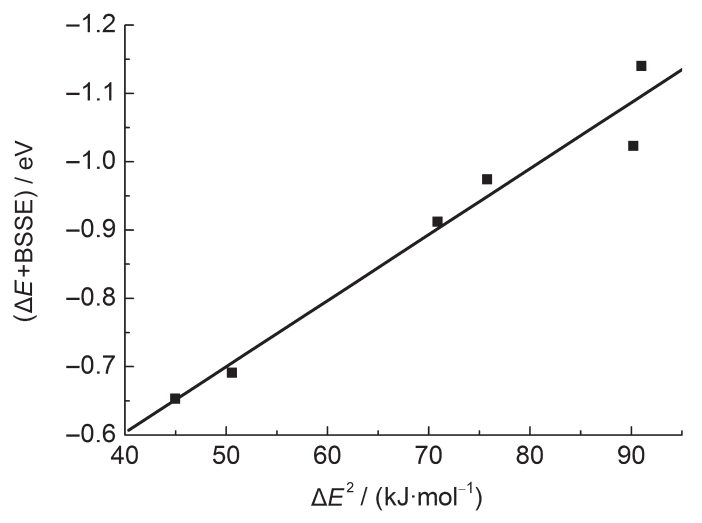

图 3 二级微扰稳定化能和相互作用能的关系

Fig.3 Relationship between second-order perturbation stabilization energy and interaction energy
相互作用中的适用性.

图 4 显示了咪唑胺客体分子与镁卟啉的 RDG 填色等值面图与 RDG 对应的 $\operatorname{sign}\left(\lambda_{2}(r)\right) \rho(r)$ 散点图. 将 $\operatorname{sign}\left(\lambda_{2}(r)\right) \rho(r)$ 函数的范围定义在 $-0.04-0.02$ a.u. 之间, 表面颜色为蓝-绿-红, 其中蓝色越深相互作用 越强, 越接近红色排斥作用越大(如空间位阻效应 越大), 中间的绿色部分体现范德华相互作用. 体系 中是否存在弱相互作用, 最主要区别在于其散点图 中最左侧区域处是否有坚条, 在文献 ${ }^{17}$ 中被称为 “spike”, 这个坚条上的点正是弱相互作用区域所对 应的点. 如图 4, 散点图中约为 -0.031 a.u. 的数值即为 等值面图中 $\mathrm{N}(42)$ 与 $\mathrm{Mg}(25)$ 所形成配位作用最强的 1 号区域, 在散点图约为 -0.017 a.u. 处和 -0.008 a.u. 处分别为 RDG 等值面图中 2 号和 3 号区的氢键和范 德华相互作用. RDG 等值面图中 2 号和 3 号区域出 现红色部分, 这源于空间位阻效应, 在散点图中接 近于 0.006 a.u.. 比较散点图和等值面图, 与 Yang 等 ${ }^{17}$ 所提出的结论较为符合, 说明 RDG 分析方法可以

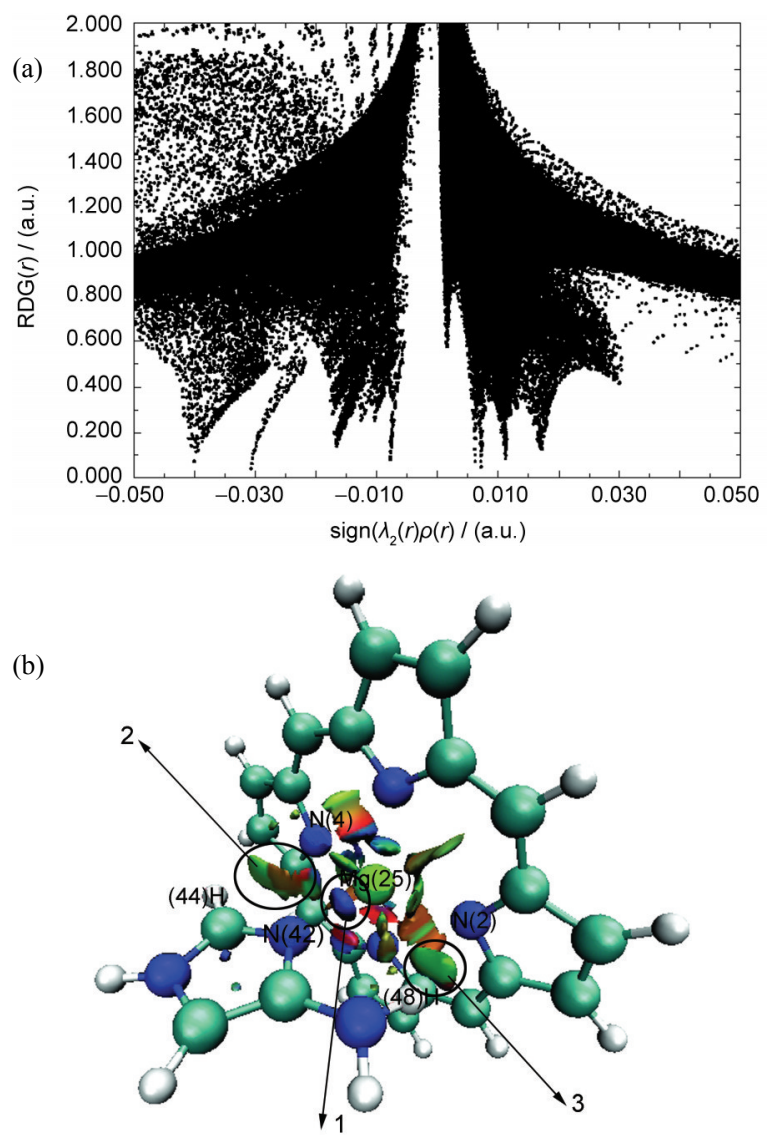

图 4 复合物 I 在 B3LYP/6-311G**水平上的散点图(a)和填 色 RDG 等值面图(b)

Fig.4 Scatter diagram (a) and color-filled RDG isosurface map (b) of complex $I$ at the $B 3 L Y P / 6-311 G$ ** level 
表 3 在 B3LYP/6-311G**水平上复合物 I-VI 和主体的概念密度泛函参数

Table 3 Conceptual DFT parameters for complexes I-VI and host at the B3LYP/6-311G** level

\begin{tabular}{|c|c|c|c|c|c|c|c|c|c|c|}
\hline \multirow{2}{*}{ Complex } & \multirow{2}{*}{$E_{\text {номо }} / \mathrm{eV}$} & \multirow{2}{*}{$E_{\text {LUмо }} / \mathrm{eV}$} & \multirow{2}{*}{$E_{\mathrm{g}} / \mathrm{eV}$} & \multirow{2}{*}{$\Delta E_{\mathrm{n}} / \mathrm{eV}$} & \multirow{2}{*}{$\Delta E_{\mathrm{c}} / \mathrm{eV}$} & \multirow{2}{*}{$\omega / \mathrm{eV}$} & \multirow{2}{*}{$\eta / \mathrm{eV}$} & \multirow{2}{*}{$\chi / \mathrm{eV}$} & \multicolumn{2}{|c|}{ NICS } \\
\hline & & & & & & & & & $\mathrm{a}$ & $\mathrm{b}$ \\
\hline host & -5.394 & -2.410 & 2.984 & 2.692 & 10.496 & 5.102 & 1.492 & 3.902 & -6.95 & -1.25 \\
\hline I & -5.000 & -2.108 & 2.892 & 2.260 & 9.369 & 4.368 & 1.446 & 3.554 & 2.44 & -5.00 \\
\hline II & -5.109 & -2.208 & 2.901 & 2.406 & 9.722 & 4.614 & 1.450 & 3.658 & 1.75 & -2.27 \\
\hline III & -5.179 & -2.303 & 2.877 & 2.562 & 10.044 & 4.865 & 1.438 & 3.741 & 26.3 & -13.9 \\
\hline IV & -5.201 & -2.266 & 2.934 & 2.484 & 9.951 & 4.751 & 1.467 & 3.734 & 6.81 & 8.37 \\
\hline V & -5.039 & -2.136 & 2.903 & 2.297 & 9.471 & 4.432 & 1.452 & 3.587 & 24.7 & -6.87 \\
\hline VI & -5.267 & -2.324 & 2.943 & 2.571 & 10.161 & 4.895 & 1.471 & 3.795 & 23.8 & 10.5 \\
\hline
\end{tabular}

$E_{\mathrm{g}}$ : energy gap defined as $E_{\text {Lимо }}$ minus $E_{\text {номо }} ; I_{\mathrm{P}}$ : ionization potential defined as $-E_{\text {номо }} ; E_{\mathrm{A}}$ : electron affinity defined $-E_{\text {LUмо }} \eta$ : absolute hardness defined as $\left(I_{\mathrm{P}}-E_{\mathrm{A}}\right) / 2 ; \chi$ : absolute electronegativity defined as $\left(I_{\mathrm{P}}+E_{\mathrm{A}}\right) / 2 ; \omega$ : electrophilicity power defined as $\chi^{2} / 2 \eta ; \Delta E_{\mathrm{e}}$ electrofugality defined as $\omega+I_{\mathrm{P}} ; \Delta E_{\mathrm{n}}$ nucleofugality defined as $\omega-E_{\mathrm{A}}$; NICS: nuclear independent chemical shift calculated at the B3LYP/6-31++G** level

很好地分析配位相互作用, 对于进一步研究镁卟啉 在生命体内的相互作用具有辅助性意义.

\section{4 概念密度泛函理论对基态电子结构分析}

表 3 列出了采用 B3LYP/6-311G**方法所得到 的主体与六个复合物概念 DFT 参数. 概念密度泛 函理论(又称密度泛函活性理论或化学密度泛函理 论) 是密度泛函理论(DFT) 的化学活性理论, 被广泛 用于研究体系的活性, ${ }^{19}$ 本文结合该参数研究形成 复合物基态结构的活性. 表中 $E_{\mathrm{g}}$ 为能隙, $\eta$ 和 $\chi$ 分别 称为绝对硬度和绝对电负性, 亲电指数 $\omega=\chi^{2} / 2 \eta$, $\Delta E_{\mathrm{n}}$ 为亲核指数差值, $\Delta E_{\mathrm{e}}$ 为亲电指数差值, 是 $\mathrm{Liu}$ 等 ${ }^{20}$ 最近提出.

由表 3 所显示数据可得, 镁卟啉主体形成复合 物后, 最高占据轨道和最低空轨道能量上升, 但总 体趋势表明复合物能隙变小. 由于复合物能隙变 小, 电子容易发生跃迁, 在电子光谱中将出现红移 现象, 说明分子间相互作用使镁卟啉主体的谱带发 生变化从而改变电信号传输, 最终影响生物体内的 诱导转换. $\eta$ 的数值用来衡量复合物的热力学稳定 性. $\eta$ 值越大, 复合物的热力学稳定性越高. $\chi$ (绝对电 负性)数值反映抗氧化性, $\chi$ 值越高, 复合物越难被氧 化. ${ }^{21}$ 六个复合物的 $\eta$ 值和 $\chi$ 值均比镁卟啉主体小, 说 明分子间相互作用的存在使复合物的热力学稳定 性和抗氧化性均比主体镁卟啉差. $\omega 、 \Delta E_{\mathrm{n}}$ 和 $\Delta E_{\mathrm{e}}$ 是预 测化学反应活性和局域选择性的有效工具. ${ }^{22}$ 表中 复合物的三个亲核/亲电指数数值均比主体小, 说明 所形成的复合物反应活性比主体高, 这与 $\eta$ 数值所 解释的热力学稳定性相一致, 即配位识别后的复合 物分子热力学稳定性下降, 反应活性升高.

\subsection{NICS 分析}

文献 ${ }^{23}$ 表明卟吩分子的芳香性来自于二氮杂
[18]轮烯和三氮杂 [18]轮烯互变结构. 同时 ${ }^{1} \mathrm{H}$ NMR 光谱数据清楚地反映出卟吩分子具有良好的共轭 体系和特殊的芳香性质. 对称性高的有机分子, 体 现其芳香性的判据很多, 如几何判据、化学判据. 本 文采用磁性判据进行芳香性分析, 即用 ${ }^{3} \mathrm{He}$ 的 NICS 值去衡量, Schleyer 等 ${ }^{24,25}$ 在相同理论方法和相同基 组计算出来的 ${ }^{3} \mathrm{He}$ 的化学位移, 和相同位置处的绝 对磁屏蔽量相似, 认为 ${ }^{3} \mathrm{He}$ 能当做一种简单而有效 的芳香性探针. 他们指出, 在环或者是笼的内部有 明显的负 NICS 值, 则显示其有横向诱导环电流存 在, 也就是说有芳香性. 反之, 具有正 NICS 值的化 合物是反芳香性的. Vaid 等 ${ }^{26}$ 应用该法计算了锂叶 啉正一价离子的芳香性, 且选取的位置为图 5 中所 示的 $a 、 b$ 两点. 本文将 ${ }^{3} \mathrm{He}$ 原子放在如图 5 中的 $a 、 b$ 两点, 用 B3LYP/6-31++G**水平计算 NICS 值 (见表 3). 从表 3 中可以看出, 主体分子在 $a 、 b$ 处的 NICS 数值均为负, 表明镁卟啉具有很好的芳香性. 六个 复合物在 $a 、 b$ 处的 NICS 数值有正有负, 其中 $a$ 点处

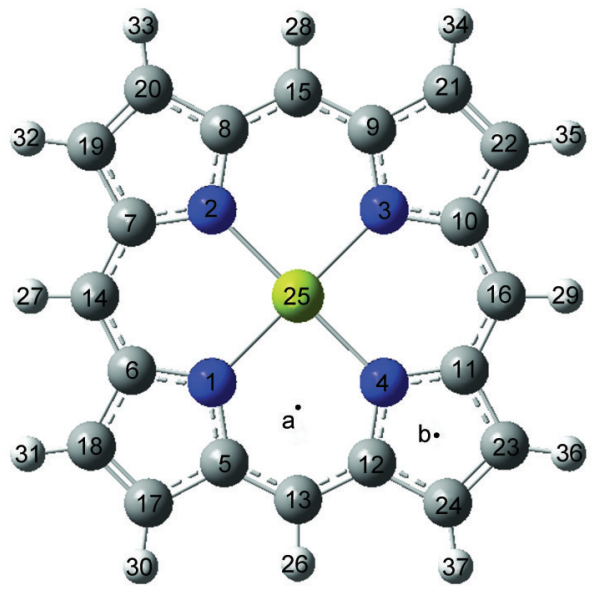

图 5 NICS 计算的位置 $a, b$

Fig.5 Locations a, b in NICS calculation 
的六个复合物数值均为正值, 说明镁卟啉主体与客 体分子相互作用后所形成的复合物在两个吡咯环 的中间即 $\mathrm{a}$ 点处无芳香性. $\mathrm{b}$ 点处 NICS 出现负值的 情况均为含氮类杂环客体与镁卟啉所形成的复合 物, 这表明含氧杂环客体对镁卟啉的作用使得复合 物中卟吩环无芳香性. 同时, 从表 3 的数据可以看 出, 含氮杂环客体与镁卟啉所形成的复合物, 在吡 咯环内 (即 b 点)不但存在芳香性而且芳香性远比主 体高, 预计产生这种现象的原因即为周边氢键作用 的影响所导致.

\section{4 结 论}

镁卟啉与氮、氧杂环客体以轴向配位作用和周 边氢键相互作用形成稳定复合物. 由于镁卟啉与 氮、氧杂环客体间的相互作用, 导致镁原子与卟吩 环不共面, 并且, 稳定化能越大, 二面角越小, 即镁 原子与叶吩环不共面程度越大. 含氮杂环比含氧杂 环客体所形成的复合物更加稳定, 这意味着在生物 体内的配位-诱导分子转换过程中含氧杂环客体容 易被含氮杂环客体所诱导从而被置换. 自然键轨道 (NBO) 分析得出氮、氧孤对电子和金属镁的空孤对 轨道即 $\mathrm{LP}(1) \mathrm{X} \rightarrow \mathrm{LP} *(1) \mathrm{Mg}$ 之间的作用对复合物的 稳定性贡献很大. 可视化 RDG 分析证明了氮、氧杂 环客体与金属镁卟啉配位作用和周边氢键相互作 用的位置、强度, 从而说明该方法可以很好地分析 配位相互作用, 对于进一步研究镁卟啉在生命体内 的相互作用具有辅助性意义. 概念密度泛函参数说 明相互作用后的六个复合物分子其热力学稳定性 和抗氧化能力均比主体分子小, 复合物的反应活性 比主体分子大, 即形成后的复合物其光电信号将会 改变, 最终影响生物体内的传递与转换. 芳香性分 析表明含氧杂环客体对镁卟啉主体的相互作用使 镁卟啉中的卟吩环呈现反芳香性; 而含氮杂环客体 使卟吩环芳香性呈现区域性, 其中两个吡咯中间(即 图 5 中 $\mathrm{a}$ 点处)呈现反芳香性, 而吡咯环处(即图 5 中 $\mathrm{b}$ 点处)比主体具有更明显的芳香性.

致谢: 本论文全部的计算工作在浙江大学宁波理工学院分 子设计与营养工程市重点实验室完成, 在此表示感谢.

\section{References}

(1) Yamamoto, Y.; Hirata, Y.; Kodama, M.; Yamaguchi, T.; Matsukawa, S.; Akiba, K.; Hashizume, D.; Lwasaki, F.;
Muranaka, A.; Uchiyama, M.; Chen, P.; Kadish, K. M.; Kobayashi, N. J. Am. Chem. Soc. 2010, 132, 12627.

(2) Mandoj, F.; Nardis, S.; Pomarico, G.; Stefanelli, M.; Schiaffino, L.; Ercolani, G.; Prodi, L.; Genovese, D.; Zaccheroni, N.; Fronczek, F. R.; Smith, K. M.; Xiao, X.; Shen, J.; Kadish, K. M.; Paolesse, R. Inorg. Chem. 2009, 48, 10346.

(3) Marques, H. M.; Brown, K. L. Coord. Chem. Rev. 2002, 225, 123.

(4) Satake, A.; Kobuke, Y. Teteahedron 2005, 61, 13.

(5) Yang, Z. W.; Yang, J.; Huang, X. J.; Tang, N.; Wu, B. Progress in Chemistry 2009, 21, 588. [杨再文, 杨 进, 黄晓卷, 唐 宁, 吴 彪. 化学进展, 2009, 21, 588.]

(6) Minaev, B.; Ågren, H. J. Chem. Phys. 2005, 315, 215.

(7) De Souza, L. A.; Da Silva, A. M., Jr.; Junqueira, G. M. A.; Carvalho, A. C. M.; Dos Santos, H. F. J. Mol. Struc. -Theochem 2010, 959, 92.

(8) Leray, I.; Vernières, M. C.; Bied-Charreton, C. Sensors and Actuators B 1999, 54, 243.

(9) Charvátová, J.; Kašička, V.; Barth, T.; Deyl, Z.; Mikšík, I.; Král, V. J. Chromatography A 2003, 1009, 73.

(10) Sobolewski, A. I.; Domcke, W. Chem. Phys. Lett. 2009, 479, 144.

(11) Zhang, P. Y.; Zhang, J. B.; Yu, X. C.; Zhang, L. W.; Wei, X. H. Acta Phys. -Chim. Sin. 2008, 24, 143. [张鹏燕, 张建斌, 于熙 昌, 张凌伟, 魏雄辉. 物理化学学报, 2008, 24, 143.]

(12) Frisch, M. J.; Trucks, G. W.; Schlegel, H. B.; et al. Gaussian 03, Revision B.01; Gaussian Inc.: Wallingford, CT, 2003.

(13) Borrelli, R.; Domcke, W. Chem. Phys. Lett. 2010, 498, 230.

(14) Boys, S. F.; Bernardi, F. Mol. Phys. 1970, 19, 553.

(15) Tian, L. Multiwfn Program, Version 2.0; http://multiwfn. codeplex.com.

(16) Zheng, X. Y.; Wang, X. Y.; Yi, S. F.; Wang, N. Q.; Peng, Y. M. J. Comput. Chem. 2010, 31, 1458.

(17) Johnson, E. R.; Keinan, S.; Mori-Sánchez, P.; Contreras-García, J.; Cohen, A. J.; Yang, W. T. J. Am. Chem. Soc. 2010, 132, 6498.

(18) Xu, L.; Sang, P.; Zou, J. W.; Xu, M. B.; Li, X. M.; Yu, Q. S. Chem. Phys. Lett. 2011, 509, 175.

(19) Liu, S. B. Acta Phys. -Chim. Sin. 2009, 25, 590. [刘述斌. 物理 化学学报, 2009, 25, 590.]

(20) Liu, S. B.; Govind, N. J. Phys. Chem. A 2008, 112, 6690.

(21) Xu, X.; Shang, Z.; Wang, G.; Li, R.; Cai, Z.; Zhao, X. J. Phys. Chem. A 2005, 109, 3754.

(22) Zhong, A. G.; Huang, L.; Li, B. L.; Jiang, H. J.; Liu, S. B. Acta Phys. -Chim. Sin. 2010, 26, 2763. [钟爱国, 黄 凌, 李佰林, 蒋华江, 刘述斌. 物理化学学报, 2010, 26, 2763.]

(23) Wang, J. J. Chin. J. Org. Chem. 2005, 25, 1353. [王进军. 有机 化学, 2005, 25, 1353.]

(24) Schleyer, P. v. R.; Jiao, H.; Glukhovtsev, M. N.; Chandrasekhar, J.; Kraka, E. J. Am. Chem. Soc. 1994, 116, 10129.

(25) Schleyer, P. v. R.; Maerker, C.; Dransfeld, A.; Jiao, H.; van Eikema Hommes, N. J. R. J. Am. Chem. Soc. 1996, 118, 6317.

(26) Cissell, J. A.; Vaid, T. P.; Yap, G. P. A. Org. Lett. 2006, 8, 2401. 